\title{
Automated Age Prediction using Wrinkles Features of Facial Images and Neural Network
}

\author{
Sudip Mandal ${ }^{1}$, Chandrima Debnath ${ }^{2}$, Lovely Kumari $^{3}$ \\ ${ }^{1}$ ECE Department, GIMT, Krishna Nagar, India \\ ${ }^{2}$ ECE Department, GIMT, Krishna Nagar, India \\ ${ }^{2}$ ECE Department, GIMT, Krishna Nagar, India
}

\begin{abstract}
Automated age estimation is an important processing task that serves many purposes such as surveillance monitoring, marketing of products, authentication systems, find out the fugitive or missing person and security control etc. Therefore, estimating age from still face images by using facial features is trending research topic from past few years. An automated age group prediction system using wrinkle features of facial images and neural network is proposed in this paper. Three age groups including child, young, and old, are considered in the classification system. The prediction process is divided into three phases: image accumulation from different website, wrinkles feature extraction using image processing technique, and age classification using Neural Network. Different facial images of different age groups are collected from several websites. The wrinkles features are extracted from each image using image processing techniques and make a corresponding database. Finally, an Artificial Neural Network (ANN) is constructed for classification of new images which will use the wrinkle features as inputs to classify the image into one of three age groups. Using this process, we can predict the age group of a face of a person with satisfactory accuracy.
\end{abstract}

Keywords: Age Prediction; Facial Feature; Wrinkles; Image Processing; Artificial Neural Network; Classification.

\section{INTRODUCTION}

Face is a prolific information source. People can effortlessly extract many kinds of useful information from a face image, such as identity, expression, emotion, gaze, gender, age, etc. Since human faces provide a lot of information, many topics have drawn lots of attentions and thus have been studied intensively. Human facial image processing has been an active and interesting research issue for years. Human being can easily predict the age of any person by looking at their facial features as we have been in the environment with them for a long time. So our brain can easily predict their approximate age but, a computer can't do it. So, it is very interesting to design an expert system which can at least estimate or predict the approximate age of a person by scanning its image automatically. Age estimation is an important processing task that serves many purposes which are given as follows.

1. In marketing, companies may increase their profits by measuring the demographics of groups interested on their billboard or street advertising through age estimation.

2. In security control and surveillance monitoring, an age estimation system, with the input of a monitoring camera, can warn or stop under-age drinkers from entering wine shops; prevent minors from purchasing tobacco products from vending machines; refuse the aged when he or she wishes to try a roller coaster at an amusement park; and deny children access to adult websites or restricted films.

3. In addition, estimated age also provides a type of soft biometric information which provides ancillary parameters for user identity. It can be used to complement primary biometric features, such as face, fingerprint, iris, and hand geometry, to improve the performance of a primary (hard) biometrics system.

4. Face-based authentication systems which typically compare age separated face images are also bound to benefit from facial ageing models and from faces.

5. It can also help to find out the fugitive or some missing person locates at remote place. 
Thus, estimating age automatically from still face images is trending research topic from past few years. A lot of approaches and models [1-8] came forward as the research progressed. The process of age estimation attempts to label a face image automatically with an age group of the individual face. Age prediction is concerned with the use of a training set to train a model that can estimate the age of the facial images. The training dataset consist of facial features for different persons with different age group. The model is any of well known artificial intelligent classifiers which can learn from the data and able to take decision.

Ageing is the process of becoming older. In the narrow sense the term refers to biological ageing of human beings, animals and other organisms. From studying the aging process of adult humans, one can observe that the facial skin of an older person is not as taut as in a younger adult or baby. These unique changes are known as the facial features based on which age of a person can be estimated. There are several face features [9-10] which are already known like geometric feature and wrinkle features. Geometric features, precisely on the basis of two-dimensional facial images. Facial characteristic points can be defined as a standard reference points on human face used by scientists. For baby the shape of face is almost circular in 2D. But as the baby grows, the distance between eyes, distance between eye and nose, distance between nose and mouth etc. are changed; consequently facial features are also changed. The second type of facial feature is adulthood. During adulthood, the main changes in this stage are changes in skin texture. Skin becomes thinner, darker, less elastic and more leathery. Facial hair become denser and change color. Also, wrinkles, under chin, sagging cheeks and lowered bags under the eyes appear. Wrinkles are a good indication of the loosening skin with respect to age (although, in general, these aging-wrinkles must not be confused with creases formed from facial expressions). Therefore, in this work, we have selected wrinkles feature as the classification parameters.

There are various popular artificial intelligence methods $[11,12]$ for classification problem such as Artificial Neural Network [13-15], Decision Tree [16-18], Bayesian Network [19-21] etc. These intelligent systems help computer to take the decision after training or learning. However, Artificial Neural Network (ANN) derives its origin from the working of human brain [22]. ANN is an information processing model which consists of multiple single processing units (neurons), these neurons are massively parallel in nature which performs highly complex computations. The sole goal of ANN is to make a computer learn something so that network would adapt to a given dataset. ANN, like people learn by example. These abilities make ANN suitable for pattern recognition, speech recognition or data classification problem. Therefore, in this work, we have chosen ANN for classification or prediction of age group.

Here, wrinkle features and Neural Network have been used to predict the age group of facial images. This paper is organized in following way. In next section, we revise some theoretical concept of some topic related with this project. Next, proposed approach and results are discussed. Finally, future work and conclusion is given followed by references.

\section{METHODOLOGY}

This automated age prediction methodology is divided into three phases: image acquisition from different website or real-life, wrinkles feature extraction using image processing technique, and age prediction using neural network.

\section{Image Acquisition}

Initially, for this project, we select the range of ages to classify them in different age groups. Following table shows three age groups (child, young, old) and corresponding range of ages for classification purpose. We have searched and saved different images with different age groups from several websites or real-life pictures. 10 images per age group are collected by this method. During acquisition of images following points must be considered.

- Facial images should be expressionless (no crying or laughing etc.). During laughing or crying, extra lines are included into the facial images. Therefore, extraction of wrinkle features from these images will be erroneous.

- The images should be makeup less as much as possible. Because if any face is covered with some makeup then the wrinkle lines may not be properly visible and extraction of wrinkle features from this images will also be erroneous. 
Sudip Mandal et al. "Automated Age Prediction Using Wrinkles Features of Facial Images and Neural Network"

- Presence of spectacles, any kind of head gadget, hats, etc. are not acceptable as they will covered a certain portion of faces and that may leads to inaccurate results.

- The faces that have hair fringes on forehead, beard on face etc. should be avoided as much as possible for the same reason.

Table1. Age groups and corresponding ages

\begin{tabular}{|l|l|l|}
\hline Age group & Range (years) & No of images \\
\hline Child & 0 to 20 & 10 \\
\hline Young & 21 to 50 & 10 \\
\hline Old & 51 and above & 10 \\
\hline
\end{tabular}

Following figures shows a sample of facial images which consists of three initial facial images for each of age groups i.e. child, young and old respectively.

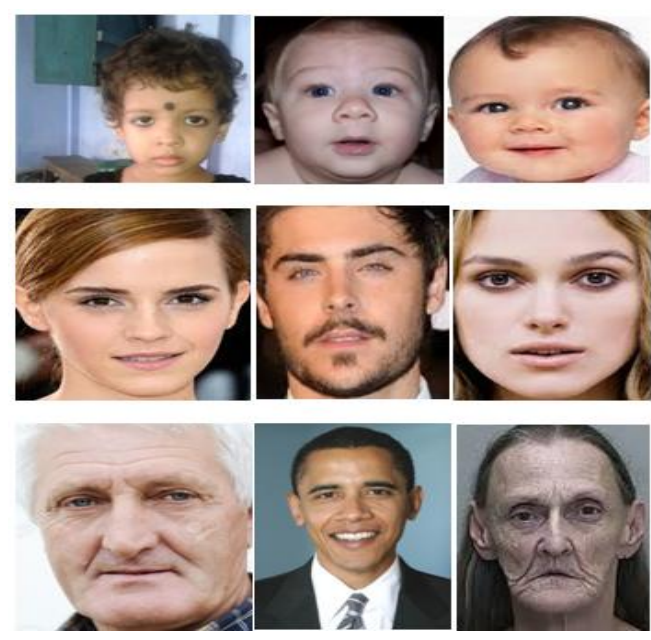

Fig1. A sample of true color images for different age groups

\section{Feature Extraction and Database Preparation}

Next for each of input true color images, the pre-processing is required i.e. to crop the face portion from a whole image. It may be done manually or some automated process using Computer Vision Toolbox. The pre-processing is required to remove unwanted portions of an image except facial portion which is area of our interest.

Now, for extraction of the wrinkle features from an image (using MATLAB), it is necessary to convert true color image into a gray scale image so that different techniques for edges detection from an image can be employed only on gray scale image. Figure 2 shows the cropped and gray scaled image of above sample images.

Sobel operator is very popular Matlab operator used for edge detection of an image. We assumed that the prominent edges of a gray scale image are corresponding to wrinkles of that face. Figure 3 denotes extracted edges or wrinkles from above mentioned samples.

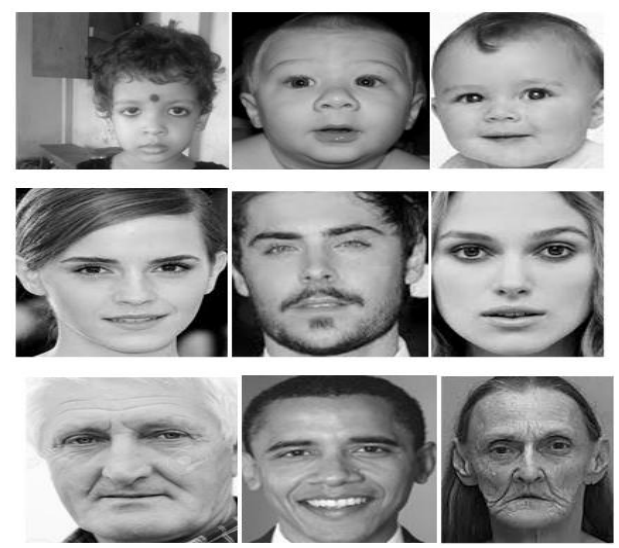

Fig2. Cropped and gray scale image of above samples 
Sudip Mandal et al. "Automated Age Prediction Using Wrinkles Features of Facial Images and Neural Network"
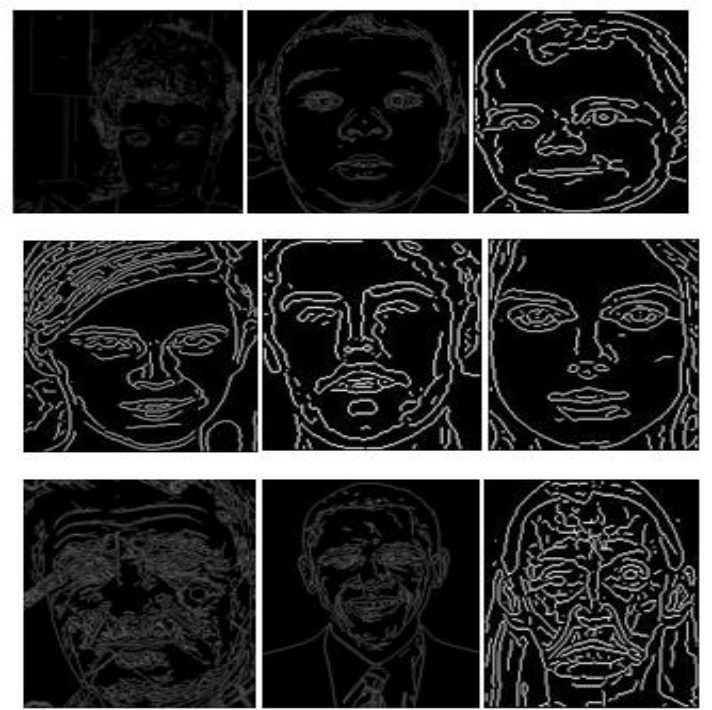

Fig3. Extracted edges or wrinkles of sample images

In this work, we only consider the wrinkle features for the classification of the different age groups. We divide a facial image into six regions like forehead, left eye, right eye, left chicks, right chicks and chin for extraction of wrinkles features. By calculating the numbers of prominent edges or wrinkles in these six different areas, six wrinkle features are calculated. Following figure shows these six different areas of a face from where numbers of different wrinkles are identified. Feature 1, Feature 2, Feature 3, Feature 4, Feature 5 and Feature 6 denote number of wrinkles in forehead, right under eye, left under eye, left under eye, right chick, left chick and chin respectively.

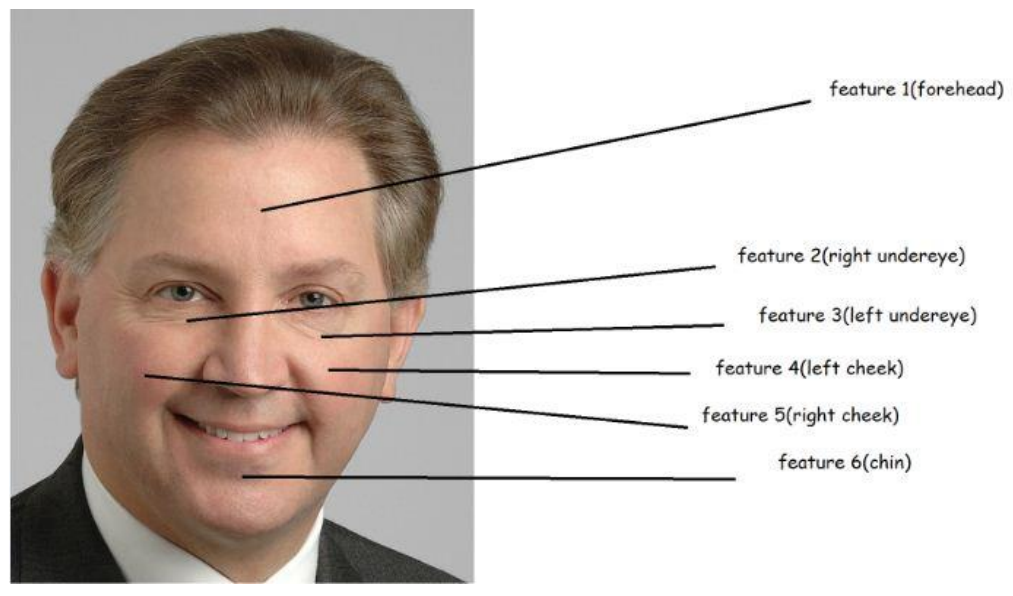

Fig4. Different wrinkle features of a facial image

Finally, we create a database to store the extracted information about number of wrinkle features and age groups. This database consists of seven columns and 30 numbers of rows i.e. total 30 facial images (10 from each age group). The first six columns denote six different wrinkle features and last column denotes age groups. Here we consider baby as 1, young as 2 and old as 3 . Following table shows a portion of the data base. This data base is used for training of Neural Network.

Table2. A sample of database consist of wrinkle features information

\begin{tabular}{|l|l|l|l|l|l|l|}
\hline F1 & F2 & F3 & F4 & F5 & F6 & Age Group \\
\hline 0 & 0 & 0 & 0 & 0 & 0 & 1 \\
\hline 0 & 0 & 0 & 0 & 0 & 0 & 1 \\
\hline 0 & 1 & 1 & 0 & 0 & 1 & 2 \\
\hline 3 & 2 & 1 & 1 & 2 & 1 & 2 \\
\hline 4 & 2 & 2 & 3 & 3 & 2 & 3 \\
\hline 10 & 3 & 3 & 4 & 4 & 6 & 3 \\
\hline
\end{tabular}


Sudip Mandal et al. "Automated Age Prediction Using Wrinkles Features of Facial Images and Neural Network"

\section{Construction of Neural Network}

In the next phase of this work, a feed forward neural network is constructed for age group classification problem which will use the wrinkle features to classify the image into any one of three age groups. The database which was created after the extraction of facial features from the images is used for training of neural network. For age classification using neural network, the six wrinkle features and age group are acted as input nodes and output node respectively.

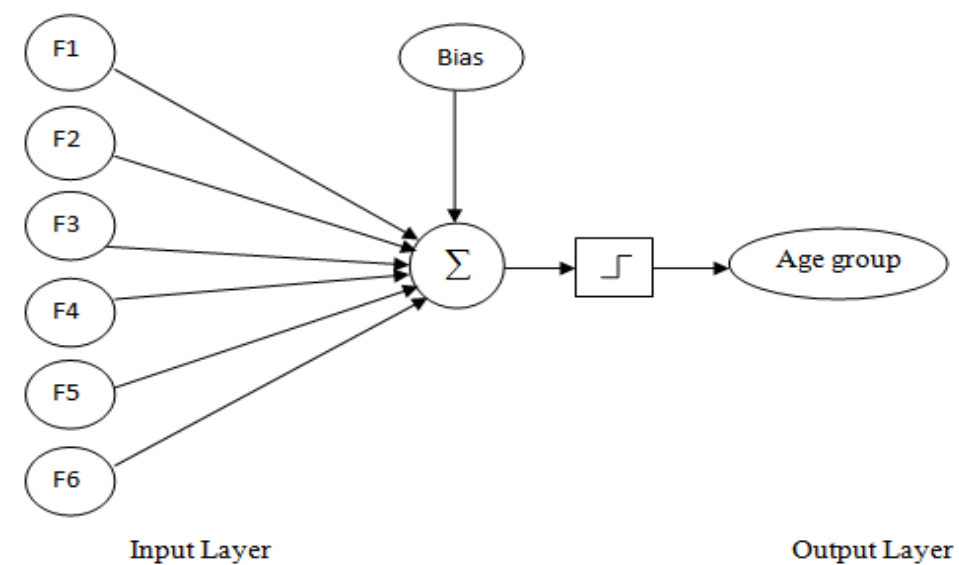

Fig5. Neural Network (Single-Layer Feed Forward) modeling of age prediction problem

We have used MATLAB 7.6 for training of Neural Network. After the training of NN, machine can learn optimal values of weights, bias, and thresholds by minimizing the training error during iteration. After training of neural network, it is necessary to validate the proposed model for age classification problem. Following is the stepwise process of the proposed approaches

- Step1: Input RGB Images

- Step2: Cropping of face portion (manually or automatically)

- Step3: Convert into Gray Scale Image

- Step4: Wrinkle features extraction using Edge Detection techniques

- Step5: Repeat step1 to step 4 for different person with different age (child, young, old) and make a database

- Step7: Use this database for training Artificial Neural Network.

- Step8: Choose new image of person and predict age group of the image using ANN model and check the accuracy.

\section{EXPERIMENTAL RESULTS AND DISCUSSION}

So, using above mention process we can identify the number of wrinkles in six different regions which are nothing bit the wrinkles features. By extracting and storing the wrinkle features and age group information, the database is generated. $90 \%$ of this database (9 images from each group i.e. 27) is used for learning of ANN structure. Rest of $10 \%$ data (1 image from each group i.e. 3 ) is remained for testing purpose. Two types of validation are performed to observe the accuracy of the model. First one is cross validation and another one is testing new cases. In case of cross validation, we apply or test the trained neural network model on training data itself i.e. trained neural network is used to predict the age group of input training images. In second case, we test the new data (which is not used during training) by trained neural network i.e. the model is used to predict the age group of new images those are taken from the internet.

\section{Neural Network with No Hidden Layer}

Initially, we have used a single-layer feed forward NN (with no hidden layer) for age prediction purpose. The classification accuracy for cross validation is $77.78 \%$. Corresponding confusion matrix for cross validation is given as 
Sudip Mandal et al. "Automated Age Prediction Using Wrinkles Features of Facial Images and Neural Network"

Table3. Confusion matrix for cross validation with single-layer feed forward NN

\begin{tabular}{|l|l|l|l|l|}
\hline Confusion Matrix & Pge Group & Predicted & Old \\
\hline \multirow{4}{*}{ Actual } & Child & Child & Young & Old \\
\cline { 2 - 5 } & Young & 8 & 1 & 0 \\
\cline { 2 - 5 } & Old & 2 & 7 & 0 \\
\hline
\end{tabular}

Accuracy for cross validation $=\frac{8+7+6}{27}=77.78 \%$

In the next phase of our work we apply the trained single-layer feed forward NN model on three new facial images which are not used as training data. The classification accuracy for testing new faces using single layer feed forward NN is $66.67 \%$.

Table4. Confusion matrix for testing new cases with single-layer feed forward NN

\begin{tabular}{|l|l|l|l|l|}
\hline Confusion Matrix & \multicolumn{2}{l|}{ Predicted } \\
\hline \multirow{4}{*}{ Actual } & Age Group & Child & Young & Old \\
\cline { 2 - 6 } & Child & 0 & 1 & 0 \\
\cline { 2 - 6 } & Young & 0 & 1 & 0 \\
\cline { 2 - 5 } & Old & 0 & 0 & 1 \\
\hline
\end{tabular}

Accuracy for classifying new images $=\frac{1+1}{3}=66.67 \%$

It can be clearly observed that the accuracy for single layer feed forward NN is not satisfactory. The reason behind it is that some wrinkles features information of (young and old) or (child and young) age group faces are similar for few cases. Moreover, wrinkles features due to ageing are somewhat person dependent i.e. they also depend on bone structure and facial expression of that person. The relationship between these wrinkles features.

It is obvious that relationships between the different wrinkles features are nonlinear and complex in nature. Therefore, accuracy for this case is not good as single-layer feed forward NN is suitable for classification of linear separable data.

\section{Neural Network with Hidden Layer(s)}

In the next phase of this work, we have tried to improve the classification accuracy by introducing nonlinearity using Multilayer Feed Forward Neural Network (MLFFNN) [23]. Here, different numbers of hidden layers with different number of nodes in it are incorporated between the input and output layer to observe the improvement in classification accuracy. It has been found that for a NN structure 2 hidden layers with two nodes on each layer is can classify the whole system with $100 \%$ accuracy for both cross validation and testing new cases. That means, MLFFNN can predict all ages correctly and it can deal with non linearity of the data.

Following figures shows multi-layer feed forward Neural Network model along with its performance.
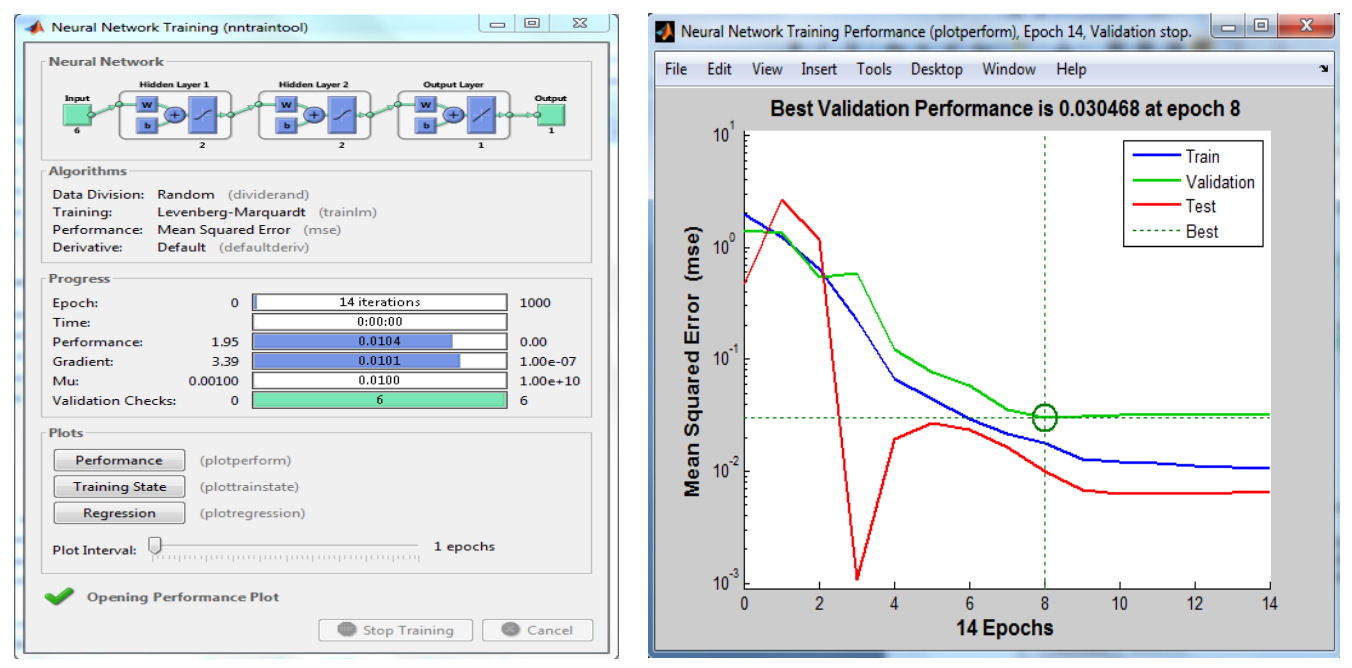

Fig5.(a). MLFFNN model for age prediction (b) Performance during training 
Sudip Mandal et al. "Automated Age Prediction Using Wrinkles Features of Facial Images and Neural Network"

Table 5 and 6 denote the confusion matrix for cross validation and testing new cases respectively with multi-layer feed forward NN.

Table 5: Confusion matrix for cross validation with multi-layer feed forward NN

\begin{tabular}{|c|c|c|c|c|}
\hline \multicolumn{2}{|c|}{ Confusion Matrix } & \multicolumn{3}{|c|}{ Predicted } \\
\hline \multirow{4}{*}{ Actual } & Age Group & Child & Young & Old \\
\hline & Child & 9 & 0 & 0 \\
\hline & Young & 0 & 9 & 0 \\
\hline & Old & 0 & 0 & 9 \\
\hline
\end{tabular}

Accuracy for cross validation with MLFFNN $=\frac{9+9+9}{27}=100 \%$

Table6. Confusion matrix for cross validation with multi-layer feed forward NN

\begin{tabular}{|l|l|l|l|l|}
\hline Confusion Matrix & Predicted & \multicolumn{2}{l|}{} \\
\hline \multirow{4}{*}{ Actual } & Age Group & Child & Young & Old \\
\cline { 2 - 5 } & Child & 1 & 0 & 0 \\
\cline { 2 - 5 } & Young & 0 & 1 & 0 \\
\cline { 2 - 5 } & Old & 0 & 0 & 1 \\
\hline
\end{tabular}

Accuracy for testing new cases with MLFFNN $=\frac{1+1+1}{3}=100 \%$

\section{CONCLUSION}

Human facial image processing has been an active and interesting research issue for years. Using facial features and artificial intelligence technique like, it is possible to predict the age group with satisfactory accuracy. In this work, we propose an automated age predication form facial images using like wrinkle feature and Neural Network which is a very popular artificial intelligence technique for classification of data.

Here, we are able to identify facial features such as wrinkles of an image by using MATLAB. Wrinkle features are extracted from the gray scale facial image using 'sobel' operator. We only consider three age groups i.e. is child, young and old. The facial images were obtained from different websites and real-life photo. The generated database for wrinkle features information is used to train the Neural Network. For age classification using neural network, the six wrinkle features and age group are acted as input nodes and output node respectively. Two types of validation technique are used: cross validation and testing new cases. Initially, single-layer feed forward NN is used. For both cases, we achieved classification accuracy slightly less. In next phase, accuracy can be improved by incorporating different number of hidden layer and nodes in the Neural Network structure. For multilayer feed forward NN, we have achieved $100 \%$ prediction accuracy for both cases.

However, our model is not robust against presence of spectacles, hats, gadgets, makeup, hair fringes and beards etc. as our proposed model based on wrinkle features only. In future, we will try to incorporate the automated face detection technique to crop the faces from a group of photo. Moreover, geometric features must be included as inputs to the Neural Network to improve the accuracy, robustness and efficiency of the proposed model.

\section{REFERENCES}

[1] H. Fukai, Y. Nishie, K.Abiko, Y. Mitsukura, M. Fukumi, and M. Tanaka, "An Age Estimation System on the AIBO", Proceedings of The International Conference on Control, Automation and Systems, pp. 2551-2554, 2008.

[2] X.Geng, Z. Zhou and K. Smith-Miles, "Automatic Age Estimation Based on Facial Aging Patterns", IEEE Transaction on Pattern Analysis And Machine Intelligence, Vol. 29, No. 12, pp. 2234-2240, 2007.

[3] X. Geng, K.Smith-Miles, and Z. Zhou, "Facial Age Estimation by Nonlinear Aging Pattern Subspace", Proceedings of the 16th ACM International Conference on Multimedia, pp. 721-724, 2008. 
Sudip Mandal et al. "Automated Age Prediction Using Wrinkles Features of Facial Images and Neural Network"

[4] W. Horng, C. Lee, and C. Chen, "Classification of Age Groups Based on Facial Features", Journal of Science and Engineering, Vol. 4, No. 3, pp. 183-192, 2001.

[5] W.-L. Chao, J.-Z. Liu, and J.-J. Ding, "Facial age estimation based on label-sensitive learning and age oriented regression", Pattern Recognition, Vol. 46, No. 3, pp. 628 - 641, 2013.

[6] S. E. Choi, Y. J. Lee, S. J. Lee, K. R. Park, and J. Kim, "Age estimation using a hierarchical classifier based on global and local facial features", Pattern Recognition, Vol. 44, No. 6, pp. 1262-1281, 2011.

[7] S. Thakur1, and L. Verma, "Identification of Face Age range Group using Neural Network", International Journal of Emerging Technology and Advanced Engineering, Vol. 2, Issue 5, pp. 250-254, 2012.

[8] M. Z. Lazarus, K. Srilakshmi and V. M. Sandeep, "Age Classification: Based On Wrinkle Analysis", International Journal on Recent and Innovation Trends in Computing and Communication, Vol. 1, Issue 3, pp. 119 - 124, 2013.

[9] E.Gose, R. Johnsonbaugh, and S. Jost, "Pattern Recognition and Image Analysis", 1st ed., Prentice Hall, Upper Saddle River, New Jersey, 1996.

[10] S. Gutta, and H. Wecheler, "Gender and ethnic classification of human faces using hybrid classifiers," Proc. Int. Joint Conference on Neural Networks, Vol. 6, pp. 4084-4089, 1999.

[11] S. Mandal, G. Saha \& R. K. Pal, "A Comparative Study on Disease Classification Using Different Soft Computing Techniques" The SIJ Transactions on Computer Science Engineering \& its Applications, Vol. 2, Issue 3, pp. 59-66, 2014.

[12] J. Han and M. Kamber, "Data Mining; Concepts and Techniques", Morgan Kaufmann Publishers, 2000.

[13] J. E. Dayhoff, "Neural-Network Architectures: An Introduction", 1st ed., Van Nostrand Reinhold Publishers, New York, 1990

[14] S. Mandal and I. Banerjee, "Cancer Classification Using Neural Network," International Journal of Emerging Engineering Research and Technology, Vol. 3, Issue 7, pp.172-178, 2015.

[15] S. Mandal, G. Saha, and R. K. Pal, "Neural Network Training Using Firefly Algorithm", Global Journal on Advancement in Engineering and Science, Vol. 1, Issue 1, pp. 07-11, 2015.

[16] F. Esposito, D. Malerba and G.i Semeraro, "A Comparative Analysis of Methods for Pruning Decision Trees", IEEE Trans. Pattern Anal. Mach. Intell, Vol. 19, 1997.

[17] W. N. Haizan, W. Mohamed, M. Najib M. Salleh, and A. H. Omar, "A Comparative Study of Reduced Error Pruning Method in Decision Tree Algorithms", Proceeding of IEEE International Conference on Control System, Computing and Engineering, pp. 23 - 25, 2012.

[18] S. Mandal, G. Saha, and R. K. Pal, "An Approach towards Automated Disease Diagnosis \& Drug Design Using Hybrid Rough-Decision Tree from Microarray Dataset", Journal of Computer Science and System Biology; Vol. 6, Issue 6; pp. 337-343, doi:10.4172/jcsb.1000130.

[19] S. Mandal, G. Saha, and R. K. Pal, "Reconstruction of Dominant Gene Regulatory Network from Microarray Data Using Rough Set and Bayesian Approach" Journal of Computer Science \& Systems Biology; Vol. 6, Issue 5, pp.262-270, 2013, doi:10.4172/jcsb.1000121.

[20] N. Friedman, and M. Goldszmidt, "Learning Bayesian networks with local structure", Kluwer Academic Publisher, pp. 421-459, 1998.

[21] N. Friedman, M. Linial, I. Nachman, and D. Pe'er, "Using Bayesian network to analyze expression data”, Journal of. Computational Biology, Vol. 7, pp. 601-620, 2000.

[22] M. Rocha, et al., "Evolution of neural networks for classification and regression", Neurocomputing, pp. 2809-2816, 2007, doi:10.1016/j.neucom.2006.05.023.

[23] D. Svozil, V. K. Eka, J. Pospichal, Introduction to multi-layer feed-forward neural networks , Chemometrics and Intelligent Laboratory Systems, vol. 39, pp. 43-62, 1997. 
Sudip Mandal et al. "Automated Age Prediction Using Wrinkles Features of Facial Images and Neural Network"

\section{AUTHORS' BIOGRAPHY}

Sudip Mandal, received M.Tech. degree in ECE from Kalyani Govt. of Engineering College. Now, he held the position of Head of Electronics and Communication Engineering Department in GIMT, Krishnanagar, India. He is also pursuing Ph.D. degree from University of Calcutta. His current research work includes Soft Computing, Computational Biology, Image Processing and Tomography. The author is also member Computational Intelligence Society and Man, System \& Cybernetics Society of IEEE. He has published 22 peer reviewed Conference and Journal paper so far.

Chandrima Debnath, is a final year student (B.Tech) of Electronics and Communication Engineering Department in GIMT, Krishnanagar, India. Her current research work includes Artificial Intelligence and Image Processing.

Lovely Kumari, is a final year student (B.Tech) of Electronics and Communication Engineering Department in GIMT, Krishnanagar, India. Her current research work includes Artificial Intelligence and Image Processing. 